lupus anticoagulant positivity, nor with single-, double- or triple- APLA positivity.

Conclusion Our data show for the first time that plasma sTREM-1 level is significantly elevated in patients with thrombotic PAPS. We suggest that soluble TREM-1 might be used as a biomarker for thrombosis in patients with primary APS and our results support the possible role for the innate immune system in the pathogenesis of thrombosis in PAPS.

\section{S3D:6 MICROANGIOPATHIC MANIFESTATIONS OF THE PRIMARY ANTIPHOSPHOLIPID SYNDROME}

J Zhao, Y Sun, Q Wang, M Li, X Zeng. Peking Union Medical College Hospital, Beijing, China

\subsection{6/lupus-2018-abstract.17}

Objective Thrombotic microangiopathy (TMA) is a relatively rare but severe vascular complication of antiphospholipid syndrome (APS) and may involve any organ. It's crucial for prompt diagnosis and early intervention, while the clinical features and outcomes of patients with TMA in APS is quite scant. The aim of this study was to investigate characteristics of primary APS with TMA patients.

Patients and methods One handred and twelve priamry APS patients who attended in Peking Union Medical College Hospital from January 2004 to December 2016 were enrolled. Patients were subdivided into two groups: PAPS with TMA and PAPS without TMA. Demographic data, clinical characteristics, laboratory features and treatment were retrospectively collected. Bivariate statistical analysis and logistical regression test were performed to compare the discrepancy between the two groups. Survival rates were studied by Kaplan-Meier method, and COX proportional hazard model was adopted to perform the analysis of predicting factors for mortality.

Results Twenty-one patients with TMA were indentified, presenting in $18.8 \%$ of all PAPS patients. Both groups were similar with respect to age of onset, gender, clinical course and pregnancy morbidity. However, the frequency of anti-b2-glycoprotein I antibodies (anti-b2GPI) $(66.7 \%$ vs $33.0 \%, \mathrm{p}=0.004)$ was significantly higher in the TMA group than the non-TMA group. In addition, thrombocytopenia (85.7\% Vs $54.9 \%$ $\mathrm{p}=0.007)$ and elevated high-sensitivity C-reactive protein (hsCRP) (71.4\% Vs 54.9\% p=0.007) were observed more frequently among TMA group. In multivariate analysis, only thrombocytopenia $(\mathrm{OR}=4.055,95 \% \mathrm{CI}: 1.006$ to 16.345 ,

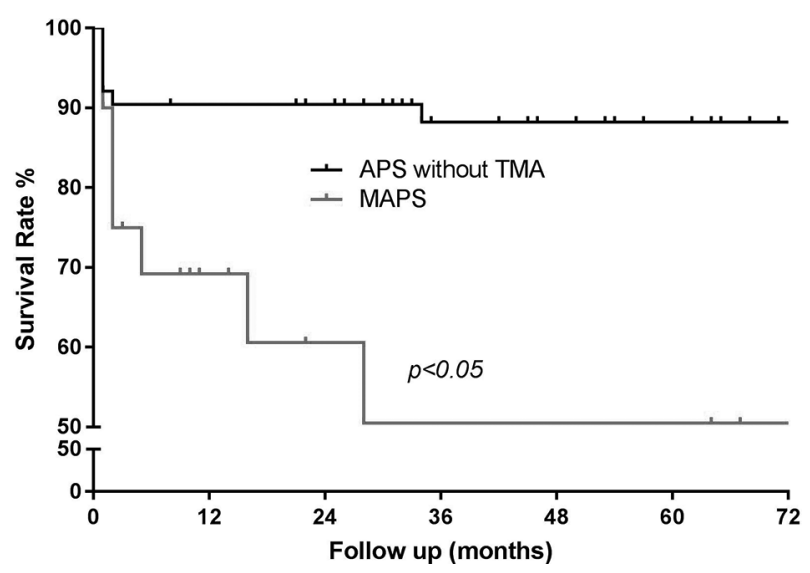

Abstract S3D:6 Figure 1 $\mathrm{p}=0.049)$, hsCRP elevation $(\mathrm{OR}=6.789,95 \% \mathrm{CI}: 2.018$ to $22.840, \mathrm{p}=0.002)$ and positivity for anti-b2GPI $(\mathrm{OR}=4.723$, 95\% CI: 1.409 to $15.830, \mathrm{p}=0.012$ ) were independent risk factors for the occurrence of TMA in PAPS patients. The overall 1, 3, and 5 year survival rate of PAPS with TMA was $69.2 \%, 60.6 \%$ and $50.5 \%$ respectively; while 90.4\%, 88.2\%, and $88.2 \%$ in APS patients without TMA $(p<0.05)$.

Conclusion Microangiopathic antiphospholipid syndromes should be an independent subset with mainly small vessels involved and worse prognosis, compared with classic APS.

\section{S3D:7 CEREBRAL HYPOPERFUSION DETECTED BY PERFUSION- WEIGHED MRI MAY ASSIST THE DIAGNOSIS OF PRIMARY DIFFUSE NEUROPSYCHIATRIC LUPUS ERYTHEMATOSUS}

${ }^{1} E$ Papadaki, ${ }^{2} \mathrm{~A}$ Fanouriakis, ${ }^{1} \mathrm{E}$ Kavroulakis, ${ }^{1} \mathrm{D}$ Karageorgou, ${ }^{3} \mathrm{P}$ Sidiropoulos, ${ }^{3} \mathrm{G}$ Bertsias, ${ }^{4} \mathrm{P}$ Simos, ${ }^{2} \mathrm{D}$ Boumpas. ${ }^{1}$ University of Crete, Department of Radiology, Heraklion, Greece; ${ }^{2}$ National and Kapodestrian University of Athens, 4th Department of Internal Medicine, Athens, Greece; ${ }^{3}$ University of Crete, Department of Rheumatology, Heraklion, Greece; ${ }^{4}$ University of Crete, Department of Psychiatry, Heraklion, Greece

\subsection{6/lupus-2018-abstract. 18}

Objective Abnormalities in regional or global cerebral perfusion have been reported in SLE but their value in distinguishing lupus from non-lupus related diffuse neuropsychiatric events has not been determined. We examined whether the addition of dynamic susceptibility-contrast-enhanced $\mathrm{T} 2 \%$ weighted perfusion MRI (DSC-MRI), a non-invasive assessment of brain haemodynamic status, to the standard MRI examination suggested by the EULAR recommendations, may be of added value in the clinical diagnosis and attribution of neuropsychiatric SLE (NPSLE).

Patients and methods Seventy-six SLE patients (53 NPSLE, 23 non-NPSLE) and 31 healthy controls underwent conventional MRI (cMRI) and DSC-MRI. Attribution of NPSLE to lupus (primary NPSLE: $n=37)$ or not $(n=16)$ was based on multidisciplinary assessment including cMRI results and response to treatment. Cerebral blood volume (CBV) and cerebral blood flow (CBF) values were estimated in 18 normal-appearing white matter (NAWM) and deep grey matter (NADGM) areas. Perfusion differences among subgroups and their diagnostic utility were assessed using Analysis of Variance, Receiver Operating Characteristics, and Binary Logistic Regression analysis.

Results The most common manifestations were mood disorder, cognitive disorder and headache. The most common manifestations were mood disorder, cognitive disorder and headache. Primary NPSLE patients had lower cerebral blood flow and volume in several NAWM areas compared to controls $(p<0.0001)$, and lower cerebral blood flow in the semioval centre bilaterally compared to non-NPSLE and non-primary NPSLE patients $(p<0.001)$. A cut-off for cerebral blood flow of 0.77 in the left semioval centre discriminated primary NPSLE from non-NPSLE/non-primary NPSLE with $80 \%$ sensitivity and 67\%-69\% specificity. Blood flow values in the left semioval centre showed substantially higher sensitivity than cMRI (81\% versus 19\%-24\%) for diagnosing primary NPSLE and the combination of the two modalities yielded $94 \%-$ $100 \%$ specificity in discriminating primary from non-primary NPSLE.

Conclusion Primary NPSLE is characterised by significant hypoperfusion in white matter and deep grey matter areas that appear normal on conventional MRI sequences. Addition 